\title{
Controllability and Observability of Fractional Linear Systems with Two Different Orders
}

\author{
Dengguo Xu, Yanmei Li, and Weifeng Zhou \\ Department of Mathematics, Chuxiong Normal University, Chuxiong, Yunnan 675000, China \\ Correspondence should be addressed to Dengguo Xu; dengguoxu@163.com
}

Received 17 September 2013; Accepted 5 December 2013; Published 20 January 2014

Academic Editors: N. Kallur and R. K. Naji

Copyright (C) 2014 Dengguo Xu et al. This is an open access article distributed under the Creative Commons Attribution License, which permits unrestricted use, distribution, and reproduction in any medium, provided the original work is properly cited.

This paper is concerned with the controllability and observability for a class of fractional linear systems with two different orders. The sufficient and necessary conditions for state controllability and state observability of such systems are established. The results obtained extend some existing results of controllability and observability for fractional dynamical systems.

\section{Introduction}

In the last three decades, interest in fractional calculus has experienced rapid growth and at present we can find many papers devoted to its theoretical and application aspects; see the work of [1] and the references therein. Fractional order models of real systems are often more adequate than the usually used integer order models in electrochemistry [2], advection dispersion models [3], anomalous diffusion [4], viscoelastic materials [5], fractal networks [6-8], robotics [9], and so forth. Further, during recent years a renewed interest has been devoted to fractional order systems in the area of automatic control; the reader can refer to monograph [10]. Oustaloup [11] initiated the first framework for noninteger order systems in the automatic control area. Fractional order control is the use of fractional calculus in the aforementioned topics, the system being modeled in a classical way or as a fractional one. From a certain point of view, the applications of fractional calculus have experienced an evolution analogous to that of control following two parallel paths depending on the starting point: the time domain or the frequency domain [12-14].

Controllability and observability are two of the most fundamental concepts in modern control theory. They have close connections to pole assignment, structural decomposition, quadratic optimal control, observer design, and so forth $[15,16]$. In the past ten years, many results have been obtained on controllability and observability of fractional order systems. Chen et al. [17] proposed robust controllability for interval fractional order linear time invariant systems, whereas Adams and Hartley [18] studied finite time controllability for fractional systems. The controllability conditions for fractional control systems with control delay were obtained in [19]. Shamardan and Moubarak [20] extended some basic results on the controllability and observability of linear discrete-time fractional order systems and developed some new concepts inherent to fractional order systems with analytical methods for checking their properties. Balachandran et al. [21] obtained controllability criteria for fractional linear systems, and then this result is extended to nonlinear fractional dynamical systems by using fixed point theorem. In recent paper [22], necessary and sufficient conditions of controllability and observability for fractional linear time invariant system are included.

However, to the best of our knowledge, there has been no result about the controllability and observability of fractional linear systems with different orders. In this paper, we investigated state controllability and state observability of fractional linear systems with two different orders. We derive the sufficient and necessary conditions on controllability and observability for the fractional linear systems with two different orders.

The paper is organized as follows. Section 2 formulates the problem and presents the preliminary results. The main results about controllability and observability for the fractional linear systems with two different orders are given in 
Sections 3 and 4, respectively. Finally, some conclusions are drawn in Section 5.

\section{Preliminaries}

Consider the following fractional linear systems with two different orders:

$$
\left[\begin{array}{c}
{ }^{c} D_{t}^{\alpha} x_{1}(t) \\
{ }^{c} D_{t}^{\beta} x_{2}(t)
\end{array}\right]=\left[\begin{array}{ll}
A_{11} & A_{12} \\
A_{21} & A_{22}
\end{array}\right]\left[\begin{array}{l}
x_{1}(t) \\
x_{2}(t)
\end{array}\right]+\left[\begin{array}{l}
B_{1} \\
B_{2}
\end{array}\right] u(t),
$$

where ${ }^{c} D_{t}^{\alpha},{ }^{c} D_{t}^{\beta}$ are the Caputo derivative, $0<\alpha<1,0<$ $\beta<1 ; x_{1} \in R^{n_{1}}, x_{2} \in R^{n_{2}}$ are the state vectors; $A_{i j} \in R^{n_{i} \times n_{j}}$, $B_{i} \in R^{n_{i} \times m}, i, j=1,2$, are the known constant matrices; $n_{1}+$ $n_{2}=n ; u \in R^{m}$ is the input vector.

When $A_{12}=A_{21}=0$, the system (1) reduces to the following form:

$$
\left[\begin{array}{c}
c \\
D_{t}^{\alpha} x_{1}(t) \\
{ }^{c} D_{t}^{\beta} x_{2}(t)
\end{array}\right]=\left[\begin{array}{cc}
A_{11} & 0 \\
0 & A_{22}
\end{array}\right]\left[\begin{array}{l}
x_{1}(t) \\
x_{2}(t)
\end{array}\right]+\left[\begin{array}{l}
B_{1} \\
B_{2}
\end{array}\right] u(t) .
$$

We first give some definitions about fractional calculus; for more details, see $[10,23,24]$.

Definition 1. Riemann-Liouville's fractional integral of order $\alpha(\alpha>0)$ for a function $h:(0, \infty) \rightarrow R$ is defined as

$$
{ }_{0} D_{t}^{-\alpha} h(t)=\frac{1}{\Gamma(\alpha)} \int_{0}^{t}(t-s)^{\alpha-1} h(s) d s
$$

where $\Gamma(\alpha)=\int_{0}^{\infty} t^{\alpha-1} e^{-t} d t$ is Gamma function.

Definition 2. Riemann-Liouville's fractional derivative of order $\alpha(0<\alpha<1)$ for a function $h:(0, \infty) \rightarrow R$ is defined as

$$
{ }_{0} D_{t}^{\alpha} h(t)=\frac{1}{\Gamma(1-\alpha)} \frac{d}{d t} \int_{0}^{t}(t-s)^{-\alpha} h(s) d s .
$$

Definition 3. The Caputo fractional derivative of order $\alpha(0<$ $\alpha<1)$ for a function $h:(0, \infty) \rightarrow R$ is defined as

$$
{ }_{0}^{c} D_{t}^{\alpha} h(t)=\frac{1}{\Gamma(1-\alpha)} \int_{0}^{t}(t-s)^{-\alpha} h^{\prime}(s) d s
$$

Throughout the paper, only the Caputo definition is used since the Laplace transform allows using initial values of classical integer order derivatives with clear physical interpretations.

According to [25], the solution of the system (1) can be obtained. Therefore, the following lemma holds.

Lemma 4. The solution of system (1) with initial conditions $x_{1}(0)=x_{10}$ and $x_{2}(0)=x_{20}$ is given by

$$
x(t)=\Phi_{0}(t) x_{0}+\int_{0}^{t} \Phi(t-\tau) B u(\tau) d \tau
$$

where

$$
\begin{aligned}
& x(t)=\left[\begin{array}{l}
x_{1}(t) \\
x_{2}(t)
\end{array}\right], \quad x_{0}=\left[\begin{array}{l}
x_{10} \\
x_{20}
\end{array}\right], \quad B=\left[\begin{array}{l}
B_{10} \\
B_{01}
\end{array}\right], \\
& B_{10}=\left[\begin{array}{c}
B_{1} \\
0
\end{array}\right], \quad B_{01}=\left[\begin{array}{c}
0 \\
B_{2}
\end{array}\right] \text {, } \\
& \Phi(t-\tau)=\left[\begin{array}{ll}
\Phi_{1}(t-\tau) & \Phi_{2}(t-\tau)
\end{array}\right] \\
& \Phi_{0}(t)=\sum_{k=0}^{\infty} \sum_{l=0}^{\infty} T_{k l} \frac{t^{k \alpha+l \beta}}{\Gamma(k \alpha+l \beta+1)}, \\
& \Phi_{1}(t)=\sum_{k=0}^{\infty} \sum_{l=0}^{\infty} T_{k l} \frac{t^{(k+1) \alpha+l \beta-1}}{\Gamma((k+1) \alpha+l \beta)}, \\
& \Phi_{2}(t)=\sum_{k=0}^{\infty} \sum_{l=0}^{\infty} T_{k l} \frac{t^{k \alpha+(l+1) \beta-1}}{\Gamma(k \alpha+(l+1) \beta)}, \\
& T_{k l}= \begin{cases}I_{n}, & k=l=0, \\
{\left[\begin{array}{cc}
A_{11} & A_{12} \\
0 & 0
\end{array}\right],} & k=1, l=0, \\
{\left[\begin{array}{cc}
0 & 0 \\
A_{21} & A_{22}
\end{array}\right],} & k=0, l=1, \\
T_{10} T_{k-1, l}+T_{01} T_{k, l-1}, & k+l>1 .\end{cases}
\end{aligned}
$$

From (8), the following lemma holds.

Lemma 5. The implication

$$
\sum_{k+l=m} T_{k l}=A^{m}, \quad m \in Z^{+}
$$

Holds, where

$$
A=\left[\begin{array}{ll}
A_{11} & A_{12} \\
A_{21} & A_{22}
\end{array}\right] .
$$

Proof. When $m=1$, it follows from (8) that

$$
\sum_{k+l=1} T_{k l}=T_{01}+T_{10}=A
$$

which implies that (9) holds when $m=1$. Now, suppose that (9) is true when $m=p, p \in Z^{+}$; namely,

$$
\sum_{k+l=p} T_{k l}=A^{p}
$$

When $m=p+1$, we get

$$
\begin{aligned}
\sum_{k+l=p+1} T_{k l} & =\sum_{k+l=p+1}\left(T_{10} T_{k-1, l}+T_{01} T_{k, l-1}\right) \\
& =\sum_{k+l=p+1} T_{10} T_{k-1, l}+\sum_{k+l=p+1} T_{01} T_{k, l-1} \\
& =\sum_{k+l=p} T_{10} T_{k, l}+\sum_{k+l=p} T_{01} T_{k, l} \\
& =T_{10} A^{p}+T_{01} A^{p} \\
& =A^{p+1}
\end{aligned}
$$


which means that (9) holds when $m=p+1$. Reasoning by mathematical induction, we can immediately conclude that (9) is true for any $m \in Z^{+}$. This therefore completes the proof.

\section{Controllability}

In this section, the sufficient and necessary conditions of controllability for the fractional linear system (1) with two different orders are discussed based on previous definitions and results. Similar to the concepts of controllability for general fractional linear systems, the definition of controllability for fractional linear systems with different orders is given as follows.

Definition 6. The system (1) is called state controllable on $[0, T]$ if given any state $x_{0}, x_{t_{1}} \in R^{n}$ there exists a control input signal $u(t):[0, T] \rightarrow R^{m}$ such that the corresponding solution of system (1) satisfies $x(0)=x_{0}$ and $x\left(t_{1}\right)=x_{t_{1}}, t_{1} \in$ $[0, T]$.

Theorem 7 . The system (1) is controllable on $\left[0, t_{1}\right]$ if and only if the controllability Gramian matrix

$$
W_{c}\left(0, t_{1}\right)=\int_{0}^{t_{1}} \Phi\left(t_{1}-\tau\right) B B^{T} \Phi^{T}\left(t_{1}-\tau\right) d \tau
$$

is nonsingular.

Proof. Suppose that the matrix $W_{c}\left(0, t_{1}\right)$ is nonsingular. Accordingly, $W_{c}\left(0, t_{1}\right)$ is invertible. Then given an initial state $x(0)=x_{0} \neq 0$, choose

$$
u(t)=B^{T} \Phi^{T}\left(t_{1}-t\right) W_{c}^{-1}\left(0, t_{1}\right)\left[x_{t_{1}}-\Phi_{0}\left(t_{1}\right) x_{0}\right]
$$

it follows from the solution of system (1) that

$$
\begin{aligned}
x\left(t_{1}\right)= & \Phi_{0}\left(t_{1}\right) x_{0}+\int_{0}^{t_{1}} \Phi\left(t_{1}-\tau\right) B u(\tau) d \tau \\
= & \Phi_{0}\left(t_{1}\right) x_{0} \\
& \quad+\int_{0}^{t_{1}} \Phi\left(t_{1}-\tau\right) B B^{T} \Phi^{T}\left(t_{1}-\tau\right) W_{c}^{-1}\left(0, t_{1}\right) \\
& \quad \times\left[x_{t_{1}}-\Phi_{0}\left(t_{1}\right) x_{0}\right] d \tau \\
= & \Phi_{0}\left(t_{1}\right) x_{0}+\int_{0}^{t_{1}} \Phi\left(t_{1}-\tau\right) B B^{T} \Phi^{T}\left(t_{1}-\tau\right) d \tau \\
& \times W_{c}^{-1}\left(0, t_{1}\right)\left[x_{t_{1}}-\Phi_{0}\left(t_{1}\right) x_{0}\right] \\
= & \Phi_{0}\left(t_{1}\right) x_{0}+W_{c}\left(0, t_{1}\right) W_{c}^{-1}\left(0, t_{1}\right)\left[x_{t_{1}}-\Phi_{0}\left(t_{1}\right) x_{0}\right] \\
= & \Phi_{0}\left(t_{1}\right) x_{0}+\left[x_{t_{1}}-\Phi_{0}\left(t_{1}\right) x_{0}\right]=x_{t_{1}} .
\end{aligned}
$$

Thus, the system (1) is controllable on $\left[0, t_{1}\right]$.
We show the converse by contradiction. Suppose that the system (1) is controllable on $\left[0, t_{1}\right]$, but the matrix $W_{c}\left(0, t_{1}\right)$ is singular. Then there exists an $n \times 1$ nonzero vector $v$ such that

$$
\begin{aligned}
0=v^{T} W_{c}\left(0, t_{1}\right) v & =\int_{0}^{t_{1}} v^{T} \Phi\left(t_{1}-\tau\right) B B^{T} \Phi^{T}\left(t_{1}-\tau\right) v d \tau \\
& =\int_{0}^{t_{1}}\left\|v^{T} \Phi\left(t_{1}-\tau\right) B\right\|^{2} d \tau,
\end{aligned}
$$

which implies

$$
v^{T} \Phi\left(t_{1}-\tau\right) B \equiv 0,
$$

for all $\tau \in\left[0, t_{1}\right]$. If (1) is controllable, there exists an input that transfers the initial $x(0)=x_{0}$ to $x\left(t_{1}\right)=0$. We choose $x_{0}=-\Phi_{0}^{-1}\left(t_{1}\right) v$; then there exists an input such that

$$
x\left(t_{1}\right)=-\Phi_{0}\left(t_{1}\right) \Phi_{0}^{-1}\left(t_{1}\right) v+\int_{0}^{t_{1}} \Phi\left(t_{1}-\tau\right) B u(\tau) d \tau=0
$$

that is,

$$
v=\int_{0}^{t_{1}} \Phi\left(t_{1}-\tau\right) B u(\tau) d \tau
$$

Its premultiplication by $v^{T}$ yields

$$
v^{T} v=\int_{0}^{t_{1}} v^{T} \Phi\left(t_{1}-\tau\right) B u(\tau) d \tau=0,
$$

which contradicts $v \neq 0$. So the matrix $W_{c}\left(0, t_{1}\right)$ is nonsingular. The proof is thus completed.

In the following, we consider the special case of systems (1) with $A_{12}=A_{21}=0$. The systems (1) are reduced to

$$
\left[\begin{array}{c}
{ }^{c} D_{t}^{\alpha} x_{1}(t) \\
{ }^{c} D_{t}^{\beta} x_{2}(t)
\end{array}\right]=\left[\begin{array}{cc}
A_{11} & 0 \\
0 & A_{22}
\end{array}\right]\left[\begin{array}{l}
x_{1}(t) \\
x_{2}(t)
\end{array}\right]+\left[\begin{array}{l}
B_{1} \\
B_{2}
\end{array}\right] u(t),
$$

which can be rewritten as the following two subsystems:

$$
\begin{aligned}
& { }^{c} D_{t}^{\alpha} x_{1}(t)=A_{11} x_{1}(t)+B_{1} u(t), \\
& { }^{c} D_{t}^{\beta} x_{2}(t)=A_{22} x_{2}(t)+B_{2} u(t) .
\end{aligned}
$$

Thus, the following corollary is true.

Corollary 8. The fractional linear system (22) is controllable on $\left[0, t_{1}\right]$ if and only if the controllability Gramian matrix

$$
\begin{aligned}
& W_{c}\left(0, t_{1}\right)=\int_{0}^{t_{1}}\left[\begin{array}{c}
\sum_{k=0}^{\infty} \frac{\left(t_{1}-\tau\right)^{(k+1) \alpha-1}}{\Gamma((k+1) \alpha)} A_{11}^{k} B_{1} \\
\sum_{l=0}^{\infty} \frac{\left(t_{1}-\tau\right)^{(l+1) \beta-1}}{\Gamma((l+1) \beta)} A_{22}^{l} B_{2}
\end{array}\right] \\
& \times\left[\begin{array}{c}
\sum_{k=0}^{\infty} \frac{\left(t_{1}-\tau\right)^{(k+1) \alpha-1}}{\Gamma((k+1) \alpha)} A_{11}^{k} B_{1} \\
\sum_{l=0}^{\infty} \frac{\left(t_{1}-\tau\right)^{(l+1) \beta-1}}{\Gamma((l+1) \beta)} A_{22}^{l} B_{2}
\end{array}\right]^{T} d \tau
\end{aligned}
$$

is nonsingular. 
Proof. When $A_{12}=A_{21}=0$, system (1) is reduced to the system (22). It follows from simple computation that

$$
T_{k l}= \begin{cases}I_{n}, & k=l=0, \\ T_{01}^{l}, & k=0, \quad l=1,2, \ldots, \\ T_{10}^{k}, & l=0, \quad k=1,2, \ldots, \\ 0_{n}, & \text { others }\end{cases}
$$

where

$$
\begin{aligned}
& T_{01}=\left[\begin{array}{cc}
0 & 0 \\
0 & A_{22}
\end{array}\right], \quad T_{10}=\left[\begin{array}{cc}
A_{11} & 0 \\
0 & 0
\end{array}\right], \\
& \Phi_{0}(t)=T_{00}+T_{01} \frac{t^{\beta}}{\Gamma(\beta+1)}+T_{02} \frac{t^{2 \beta}}{\Gamma(2 \beta+1)}+\cdots \\
& +T_{10} \frac{t^{\alpha}}{\Gamma(\alpha+1)}+T_{20} \frac{t^{2 \alpha}}{\Gamma(2 \alpha+1)}+\cdots \\
& =\left[\begin{array}{cc}
I_{n_{1}} & 0 \\
0 & I_{n_{2}}
\end{array}\right]+\frac{t^{\beta}}{\Gamma(\beta+1)}\left[\begin{array}{cc}
0 & 0 \\
0 & A_{22}
\end{array}\right] \\
& +\frac{t^{2 \beta}}{\Gamma(2 \beta+1)}\left[\begin{array}{cc}
0 & 0 \\
0 & A_{22}
\end{array}\right]^{2}+\cdots \\
& +\frac{t^{\alpha}}{\Gamma(\alpha+1)}\left[\begin{array}{cc}
A_{11} & 0 \\
0 & 0
\end{array}\right] \\
& +\frac{t^{2 \alpha}}{\Gamma(2 \alpha+1)}\left[\begin{array}{cc}
A_{11} & 0 \\
0 & 0
\end{array}\right]^{2}+\cdots \\
& =\left[\begin{array}{cc}
\sum_{k=0}^{\infty} \frac{t^{k \alpha}}{\Gamma(k \alpha+1)} A_{11}^{k} & 0 \\
0 & \sum_{l=0}^{\infty} \frac{t^{l \beta}}{\Gamma(l \beta+1)} A_{22}^{l}
\end{array}\right], \\
& \Phi_{1}(t)=T_{00} \frac{t^{\alpha-1}}{\Gamma(\alpha)}+T_{01} \frac{t^{\alpha+\beta-1}}{\Gamma(\alpha+\beta)}+T_{02} \frac{t^{\alpha+2 \beta-1}}{\gamma(\alpha+2 \beta)}+\cdots \\
& +T_{10} \frac{t^{2 \alpha-1}}{\Gamma(2 \alpha)}+T_{20} \frac{t^{3 \alpha-1}}{\Gamma(3 \alpha)}+\cdots \\
& =\frac{t^{\alpha-1}}{\Gamma(\alpha)}\left[\begin{array}{cc}
I_{n_{1}} & 0 \\
0 & I_{n_{2}}
\end{array}\right]+\frac{t^{\alpha+\beta-1}}{\Gamma(\alpha+\beta)}\left[\begin{array}{cc}
0 & 0 \\
0 & A_{22}
\end{array}\right] \\
& +\frac{t^{\alpha+2 \beta-1}}{\Gamma(\alpha+2 \beta)}\left[\begin{array}{cc}
0 & 0 \\
0 & A_{22}
\end{array}\right]^{2}+\cdots \\
& +\frac{t^{2 \alpha-1}}{\Gamma(2 \alpha)}\left[\begin{array}{cc}
A_{11} & 0 \\
0 & 0
\end{array}\right]
\end{aligned}
$$

$$
\begin{aligned}
& +\frac{t^{3 \alpha-1}}{\Gamma(3 \alpha)}\left[\begin{array}{cc}
A_{11} & 0 \\
0 & 0
\end{array}\right]^{2}+\cdots \\
& =\left[\begin{array}{cc}
\sum_{k=0}^{\infty} \frac{t^{(k+1) \alpha-1}}{\Gamma((k+1) \alpha)} A_{11}^{k} & 0 \\
0 & \sum_{l=0}^{\infty} \frac{t^{\alpha+l \beta-1}}{\Gamma(\alpha+l \beta)} A_{22}^{l}
\end{array}\right], \\
& \Phi_{2}(t)=T_{00} \frac{t^{\beta-1}}{\Gamma(\beta)}+T_{01} \frac{t^{2 \beta-1}}{\Gamma(2 \beta)}+T_{02} \frac{t^{3 \beta-1}}{\Gamma(3 \beta)}+\cdots \\
& +T_{10} \frac{t^{\alpha+\beta-1}}{\Gamma(\alpha+\beta)}+T_{20} \frac{t^{2 \alpha+\beta-1}}{\Gamma(2 \alpha+\beta)}+\cdots \\
& =\frac{t^{\beta-1}}{\Gamma(\beta)}\left[\begin{array}{cc}
I_{n_{1}} & 0 \\
0 & I_{n_{2}}
\end{array}\right]+\frac{t^{2 \beta-1}}{\Gamma(2 \beta)}\left[\begin{array}{cc}
0 & 0 \\
0 & A_{22}
\end{array}\right] \\
& +\frac{t^{3 \beta-1}}{\Gamma(3 \beta)}\left[\begin{array}{cc}
0 & 0 \\
0 & A_{22}
\end{array}\right]^{2}+\cdots \\
& +\frac{t^{\alpha+\beta-1}}{\Gamma(\alpha+\beta)}\left[\begin{array}{cc}
A_{11} & 0 \\
0 & 0
\end{array}\right]+\frac{t^{2 \alpha+\beta-1}}{\Gamma(2 \alpha+\beta)}\left[\begin{array}{cc}
A_{11} & 0 \\
0 & 0
\end{array}\right]^{2}+\cdots \\
& =\left[\begin{array}{cc}
\sum_{k=0}^{\infty} \frac{t^{k \alpha+\beta-1}}{\Gamma(k \alpha+\beta)} A_{11}^{k} & 0 \\
0 & \sum_{l=0}^{\infty} \frac{t^{(l+1) \beta-1}}{\Gamma((l+1) \beta)} A_{22}^{l}
\end{array}\right] \text {. }
\end{aligned}
$$

Therefore, the controllability Gramian matrix in the Theorem 7 is reduced to

$$
\begin{aligned}
& W_{c}\left(0, t_{1}\right)=\int_{0}^{t_{1}}\left[\begin{array}{l}
\sum_{k=0}^{\infty} \frac{\left(t_{1}-\tau\right)^{(k+1) \alpha-1}}{\Gamma((k+1) \alpha)} A_{11}^{k} B_{1} \\
\sum_{l=0}^{\infty} \frac{\left(t_{1}-\tau\right)^{(l+1) \beta-1}}{\Gamma((l+1) \beta)} A_{22}^{l} B_{2}
\end{array}\right] \\
& \times\left[\begin{array}{c}
\sum_{k=0}^{\infty} \frac{\left(t_{1}-\tau\right)^{(k+1) \alpha-1}}{\Gamma((k+1) \alpha)} A_{11}^{k} B_{1} \\
\sum_{l=0}^{\infty} \frac{\left(t_{1}-\tau\right)^{(l+1) \beta-1}}{\Gamma((l+1) \beta)} A_{22}^{l} B_{2}
\end{array}\right]^{T} d \tau \text {. }
\end{aligned}
$$

This completes the proof.

Obviously, the following proposition is true.

Proposition 9. The fractional linear system (22) is controllable if and only if subsystems (23a) and (23b) are all controllable.

In the following, we consider another special case of system (1). When $\alpha=\beta$ in the system (1), it is reduced to

$$
{ }^{c} D_{t}^{\alpha} x(t)=A x(t)+B u(t)
$$


where

$$
x(t)=\left[\begin{array}{l}
x_{1}(t) \\
x_{2}(t)
\end{array}\right], \quad A=\left[\begin{array}{ll}
A_{11} & A_{12} \\
A_{21} & A_{22}
\end{array}\right], \quad B=\left[\begin{array}{l}
B_{1} \\
B_{2}
\end{array}\right] .
$$

Corollary 10. The fractional linear system (28) is controllable on $\left[0, t_{1}\right]$ if and only if the controllability Gramian matrix

$$
\begin{aligned}
W_{c}=\int_{0}^{t_{1}} & \sum_{k=0}^{\infty} \frac{\left(t_{1}-\tau\right)^{(k+1) \alpha-1}}{\Gamma((k+1) \alpha)} A^{k} B B^{T} \\
& \times \sum_{K=0}^{\infty} \frac{\left(t_{1}-\tau\right)^{(k+1) \alpha-1}}{\Gamma((k+1) \alpha)}\left(A^{T}\right)^{k} d \tau
\end{aligned}
$$

is nonsingular.

Proof. According to the result of Lemma 5, when $\alpha=\beta$, we can obtain

$$
\begin{aligned}
& \Phi_{0}(t)=\sum_{k=0}^{\infty}\left(T_{k 0} \frac{t^{k \alpha}}{\Gamma(k \alpha+1)}+T_{k 1} \frac{t^{(k+1) \alpha}}{\Gamma((k+1) \alpha+1)}+\cdots\right) \\
& =T_{00}+T_{01} \frac{t^{\alpha}}{\Gamma(\alpha+1)}+T_{02} \frac{t^{2 \alpha}}{\Gamma(2 \alpha+1)} \\
& +T_{03} \frac{t^{3 \alpha}}{\Gamma(3 \alpha+1)}+\cdots+T_{10} \frac{t^{\alpha}}{\Gamma(\alpha+1)} \\
& +T_{11} \frac{t^{2 \alpha}}{\Gamma(2 \alpha+1)}+T_{12} \frac{t^{3 \alpha}}{\Gamma(3 \alpha+1)}+\cdots \\
& +T_{20} \frac{t^{2 \alpha}}{\Gamma(2 \alpha+1)}+T_{21} \frac{t^{3 \alpha}}{\Gamma(3 \alpha+1)} \\
& +T_{22} \frac{t^{4 \alpha}}{\Gamma(4 \alpha+1)}+\cdots+T_{30} \frac{t^{3 \alpha}}{\Gamma(3 \alpha+1)} \\
& +T_{31} \frac{t^{4 \alpha}}{\Gamma(4 \alpha+1)}+T_{32} \frac{t^{5 \alpha}}{\Gamma(5 \alpha+1)}+\cdots \\
& =T_{00}+\frac{t^{\alpha}}{\Gamma(\alpha+1)}\left(T_{01}+T_{10}\right) \\
& +\frac{t^{2 \alpha}}{\Gamma(2 \alpha+1)}\left(T_{02}+T_{11}+T_{20}\right) \\
& +\frac{t^{3 \alpha}}{\Gamma(3 \alpha+1)}\left(T_{03}+T_{12}+T_{21}+T_{30}\right)+\cdots \\
& =\left[\begin{array}{cc}
I_{n 1} & 0 \\
0 & I_{n 2}
\end{array}\right]+\frac{t^{\alpha}}{\Gamma(\alpha+1)} A+\frac{t^{2 \alpha}}{\Gamma(2 \alpha+1)} A^{2} \\
& +\frac{t^{3 \alpha}}{\Gamma(3 \alpha+1)} A^{3}+\cdots=\sum_{k=0}^{\infty} \frac{t^{k \alpha}}{\Gamma(k \alpha+1)} A^{k} .
\end{aligned}
$$

For the same reason as before, we get

$$
\begin{aligned}
\Phi_{1}(t)=\Phi_{2}(t) & =\sum_{k=0}^{\infty} \sum_{l=0}^{\infty} T_{k l} \frac{t^{(k+l+1) \alpha-1}}{\Gamma((k+l+1) \alpha)} \\
& =\sum_{k=0}^{\infty} \frac{t^{(k+1) \alpha-1}}{\Gamma((k+1) \alpha)} A^{k} .
\end{aligned}
$$

Therefore, by simple computation, the controllability Gramian matrix of the system (28) can be obtained as (30). The proof is thus completed.

Remark 11. Corollary 10 is equivalent to the result of Theorem 2.2 in [21]. Therefore, Theorem 7 of this paper extends the existing results to a more general case.

\section{Observability}

In this section, we treat another fundamental property of the fractional linear system with different orders, namely, observability with respect to a linear output. Throughout the rest of this paper, we consider the system (1) with the following output equation:

$$
\left[\begin{array}{l}
y_{1}(t) \\
y_{2}(t)
\end{array}\right]=\left[\begin{array}{ll}
C_{11} & C_{12} \\
C_{21} & C_{22}
\end{array}\right]\left[\begin{array}{l}
x_{1}(t) \\
x_{2}(t)
\end{array}\right]
$$

where $y_{1} \in R^{p_{1}}, y_{2} \in R^{p_{2}}$ are the output vectors; $x_{1} \in$ $R^{n_{1}}, x_{2} \in R^{n_{2}}$ are the state vectors in the system (1); $C_{i j} \in$ $R^{p_{i} \times n_{j}}, \quad i, j=1,2$, are the known constant matrices; $p_{1}+p_{2}=$ p.

When $C_{12}=C_{21}=0$, the output (33) is reduced to the following simple form:

$$
\left[\begin{array}{l}
y_{1}(t) \\
y_{2}(t)
\end{array}\right]=\left[\begin{array}{cc}
C_{11} & 0 \\
0 & C_{22}
\end{array}\right]\left[\begin{array}{l}
x_{1}(t) \\
x_{2}(t)
\end{array}\right],
$$

which is equivalent to two suboutput equations as follows:

$$
\begin{aligned}
& y_{1}=C_{11} x_{1}, \\
& y_{2}=C_{22} x_{2} .
\end{aligned}
$$

Definition 12. The system (1) with the output (33) are called state observable on $[0, T]$ if any initial state $x(0)=x_{0} \in R^{n}$ can be uniquely determined by the corresponding system input $u(t)$ and system output $y(t)$, for $t \in[0, T]$.

Define observability Gramian matrix $W_{o}(0, t)$ as

$$
W_{o}(0, t)=\int_{0}^{t} \Phi_{0}^{T}(\tau) C^{T} C \Phi_{0}(\tau) d \tau
$$

where

$$
C=\left[\begin{array}{ll}
C_{11} & C_{12} \\
C_{21} & C_{22}
\end{array}\right]
$$

Theorem 13. The system (1) with the output (33) is observable on $\left[0, t_{1}\right]$ if and only if the observability Gramian matrix

$$
W_{o}\left(0, t_{1}\right)=\int_{0}^{t_{1}} \Phi_{0}^{T}(\tau) C^{T} C \Phi_{0}(\tau) d \tau
$$

is invertible. 
Proof. It follows from Lemma 4 that the output of system (1) has the following expression:

$$
\begin{aligned}
y(t) & =C x(t) \\
& =C \Phi_{0}(t) x_{0}+C \int_{0}^{t} \Phi(t-\tau) B u(\tau) d \tau .
\end{aligned}
$$

It is easy to see from Definition 12 that the observability of system (1) is equivalent to the observability of $y(t)$ given by

$$
y(t)=C \Phi_{0}(t) x_{0}
$$

as $u(t)=0$.

Multiplying both sides of (40) by $\Phi_{0}^{T}(t) C^{T}$, and integrating with respect to $t$ from 0 to $t_{1}$, we have

$$
\int_{0}^{t_{1}} \Phi_{0}^{T}(t) C^{T} y(t) d t=W_{o}\left(0, t_{1}\right) x_{0} .
$$

Obviously, the left-hand side of (41) depends on $y(t)$, and the right-hand side in (41) does not depend on $y(t), t \in\left[0, t_{1}\right]$. Thus, (41) is a linear algebraic equation of $x_{0}$. If $W_{o}\left(0, t_{1}\right)$ is invertible, then the initial state $x(0)=x_{0}$ is uniquely determined by the corresponding system output $y(t)$, for $t \epsilon$ $\left[0, t_{1}\right]$. Namely, the system $(1)$ is observable on $\left[0, t_{1}\right]$.

Next we show that if $W_{o}\left(0, t_{1}\right)$ is singular for all $t_{1}$, then system (1) with the output (33) is not observable. Suppose $W_{o}\left(0, t_{1}\right)$ is singular; then there exists an $n \times 1$ nonzero constant vector $v$ such that

$$
\begin{aligned}
v^{T} W_{o}\left(0, t_{1}\right) v & =\int_{0}^{t_{1}} v^{T} \Phi_{0}^{T}(\tau) C^{T} C \Phi_{0}(\tau) v d \tau \\
& =\int_{0}^{t_{1}}\left\|C \Phi_{0}(\tau) v\right\|^{2} d \tau=0,
\end{aligned}
$$

which implies that

$$
C \Phi_{0}(\tau) v \equiv 0
$$

for all $\tau \in\left[0, t_{1}\right]$. If we choose $x(0)=x_{0}=v$, then the output (33) is given by

$$
y(t)=C \Phi_{0}(t) x_{0}=C \Phi_{0}(t) v \equiv 0
$$

Thus, the initial state $x_{0}$ cannot be uniquely determined by $y(t)$. Therefore, the system (1) with the output (33) is not observable. This completes the proof.
Remark 14. When $\alpha=\beta$ in the system $(1), \Phi_{0}(t)$ is already obtained in the proof of Corollary 10. Therefore, the observability Gramian matrix in Theorem 13 is

$$
\begin{aligned}
W_{o}\left(0, t_{1}\right) & =\int_{0}^{t_{1}} \Phi_{0}^{T}(\tau) C^{T} C \Phi_{0}(\tau) d \tau \\
& =\int_{0}^{t_{1}} \sum_{k=0}^{\infty} \frac{\tau^{k \alpha}}{\Gamma(k \alpha+1)}\left(A^{T}\right)^{k} C^{T} C \sum_{k=0}^{\infty} \frac{\tau^{k \alpha}}{\Gamma(k \alpha+1)} A^{k} d \tau
\end{aligned}
$$

which is the observability Gramian matrix in paper [22] by denoting $E_{\alpha}\left(A t^{\alpha}\right)=\sum_{k=0}^{\infty}\left(\tau^{k \alpha} / \Gamma(k \alpha+1)\right) A^{k}$. Therefore, Theorem 13 is actually a generalization of the existing observability results for the fractional linear system.

When $A_{12}=A_{21}=0$ and $C_{12}=C_{21}=0$, the system (1) with the output (33) is reduced to the following state equation and output equation:

$$
\begin{gathered}
{\left[\begin{array}{c}
c \\
{ }^{c} D_{t}^{\alpha} x_{1}(t) \\
{ }^{\beta} x_{2}(t)
\end{array}\right]=\left[\begin{array}{cc}
A_{11} & 0 \\
0 & A_{22}
\end{array}\right]\left[\begin{array}{l}
x_{1}(t) \\
x_{2}(t)
\end{array}\right]+\left[\begin{array}{l}
B_{1} \\
B_{2}
\end{array}\right] u(t),} \\
{\left[\begin{array}{l}
y_{1}(t) \\
y_{2}(t)
\end{array}\right]=\left[\begin{array}{cc}
C_{11} & 0 \\
0 & C_{22}
\end{array}\right]\left[\begin{array}{l}
x_{1}(t) \\
x_{2}(t)
\end{array}\right]}
\end{gathered}
$$

which can be rewritten as the following two subsystems with input and output:

$$
\begin{gathered}
{ }^{c} D_{t}^{\alpha} x_{1}(t)=A_{11} x_{1}(t)+B_{1} u(t), \\
y_{1}(t)=C_{11} x_{1}(t),
\end{gathered}
$$

$$
\begin{gathered}
{ }^{c} D_{t}^{\beta} x_{1}(t)=A_{22} x_{2}(t)+B_{2} u(t), \\
y_{2}(t)=C_{22} x_{2}(t) .
\end{gathered}
$$

It follows from conditions $A_{12}=A_{21}=0$ and $C_{12}=C_{21}=0$ that

$$
\begin{aligned}
& \Phi_{0}^{T}(t) C^{T} C \Phi_{0}(t) \\
& =\left[\begin{array}{cc}
\sum_{k=0}^{\infty} \frac{t^{k \alpha}}{\Gamma(k \alpha+1)} A_{11}^{k} & 0 \\
0 & \sum_{l=0}^{\infty} \frac{t^{l \beta}}{\Gamma(l \beta+1)} A_{22}^{l}
\end{array}\right]^{T}\left[\begin{array}{cc}
C_{11} & 0 \\
0 & C_{22}
\end{array}\right]^{T}\left[\begin{array}{cc}
C_{11} & 0 \\
0 & C_{22}
\end{array}\right]\left[\begin{array}{cc}
\sum_{k=0}^{\infty} \frac{t^{k \alpha}}{\Gamma(k \alpha+1)} A_{11}^{k} & 0 \\
0 & \sum_{l=0}^{\infty} \frac{t^{l \beta}}{\Gamma(l \beta+1)} A_{22}^{l}
\end{array}\right]
\end{aligned}
$$




$$
\begin{aligned}
& =\left[\begin{array}{cc}
\sum_{k=0}^{\infty} \frac{t^{k \alpha}}{\Gamma(k \alpha+1)}\left(A_{11}^{k}\right)^{T} \times C_{11}^{T} C_{11} \times \sum_{k=0}^{\infty} \frac{t^{k \alpha}}{\Gamma(k \alpha+1)} A_{11}^{k} & 0 \\
0 & \sum_{l=0}^{\infty} \frac{t^{l \beta}}{\Gamma(l \beta+1)}\left(A_{22}^{l}\right)^{T} \times C_{22}^{T} C_{22} \times \sum_{l=0}^{\infty} \frac{t^{l \beta}}{\Gamma(l \beta+1)} A_{22}^{l}
\end{array}\right] \\
& =\left[\begin{array}{cc}
E_{\alpha}\left(A_{11}^{T} t^{\alpha}\right) C_{11}^{T} \times C_{11} E_{\alpha}\left(A_{11} t^{\alpha}\right) & 0 \\
0 & E_{\beta}\left(A_{22}^{T} t^{\beta}\right) C_{22}^{T} \times C_{22} E_{\beta}\left(A_{22} t^{\beta}\right)
\end{array}\right],
\end{aligned}
$$

where $E_{s}\left(A t^{s}\right)=\sum_{k=0}^{\infty}\left(t^{k s} / \Gamma(k s+1)\right) A^{k}$ is Mittag-Leffler function.

Therefore, the following corollary holds.

Corollary 15. Denote $E_{\alpha}\left(A_{11}, t\right)=E_{\alpha}\left(A_{11}^{T} t^{\alpha}\right) C_{11}^{T} \times$ $C_{11} E_{\alpha}\left(A_{11} t^{\alpha}\right)$ and $E_{\beta}\left(A_{22}, t\right)=E_{\beta}\left(A_{22}^{T} t^{\beta}\right) C_{22}^{T} \times$ $C_{22} E_{\beta}\left(A_{22} t^{\beta}\right)$. Then the system (46a) with the output (46b) is observable on $\left[0, t_{1}\right]$ if and only if the observability Gramian matrix

$$
\int_{0}^{t_{1}}\left[\begin{array}{cc}
E_{\alpha}\left(A_{11}, t\right) & 0 \\
0 & E_{\beta}\left(A_{22}, t\right)
\end{array}\right] d t
$$

is nonsingular.

The following proposition is also true.

Proposition 16. The fractional linear system (46a) with the output (46b) is observable if and only if the fractional linear subsystems (47a) with the output (47b) and (48a) with the output (48b) are all observable.

\section{Conclusions}

In this paper, the controllability and observability problems for fractional linear systems with two different orders have been studied. The sufficient and necessary conditions for state controllability and state observability of such systems are established. The results obtained will be useful in the analysis and synthesis of fractional dynamical systems. Extending the results of this paper toward fractional linear systems consisting of $n$ subsystems with different orders is a future work.

\section{Conflict of Interests}

The authors declare that there is no conflict of interests regarding the publication of this paper.

\section{Acknowledgments}

The author would like to thank the editors and reviewers for their helpful suggestions. The present work was supported by Natural Science Foundation of Yunnan province of China (no. 2012FB175) and Key Projects of Scientific Research Funds of educational bureau of Yunnan province of China (no. 2010Z002).

\section{References}

[1] J. T. Machado, V. Kiryakova, and F. Mainardi, "Recent history of fractional calculus," Communications in Nonlinear Science and Numerical Simulation, vol. 16, no. 3, pp. 1140-1153, 2011.

[2] M. Ichise, Y. Nagayanagi, and T. Kojima, "An analog simulation of non-integer, order transfer functions for analysis of electrode ppocesses," Journal of Electroanalytical Chemistry, vol. 33, no. 2, pp. 253-265, 1971.

[3] D. A. Benson, S. W. Wheatcraft, and M. M. Meerschaert, "Application of a fractional advection-dispersion equation," Water Resources Research, vol. 36, no. 6, pp. 1403-1412, 2000.

[4] R. Metzler and J. Klafter, "The random walk's guide to anomalous diffusion: a fractional dynamics approach," Physics Report, vol. 339, no. 1, pp. 1-77, 2000.

[5] M. Renardy, W. J. Hrusa, and J. A. Nohel, Mathematical Problems in Viscoelasticity, vol. 35 of Pitman Monographs and Surveys in Pure and Applied Mathematics, Longman Scientific \& Technical, Harlow, UK, 1987.

[6] M. Al-Akaidi, Fractal Speech Processing, Cambridge University Press, 2004.

[7] P. Arena, R. Caponetto, L. Fortuna, and D. Porto, Nonlinear Noninteger Order Circuits and Systems: An Introduction, vol. 38 of World Scientific Series on Nonlinear Science Series A, World Scientific Publishing, 2000.

[8] B. J. West, M. Bologna, and P. Grigolini, Physics of Fractal Operators, Springer, New York, NY, USA, 2003.

[9] D. Valerio and J. Sa da Costa, "Non-integer order control of a flexible robot," in Proceedings of the IFAC Workshop on Fractional Differentiation and Its Applications (FDA '04), Bordeaux, France, 2004.

[10] C. A. Monje, Y. Chen, B. M. Vinagre, D. Xue, and V. FeliuBatlle, Fractional-Order Systems and Controls: Fundamentals and Applications, Springer, 2010.

[11] A. Oustaloup, La Commande CRONE: Commande Robuste d'Ordre Non Entier, Hermes, Paris, France, 1991.

[12] D. Matignon and B. Dandrea-Novel, "Some results on controllability and observability of finite-dimensional fractional differential systems," in Computational Engineering in Systems Applications, vol. 2, pp. 952-956, 1996.

[13] J. A. T. Machado, "Analysis and design of fractional-order digital control systems," Systems Analysis Modelling Simulation, vol. 27, no. 2-3, pp. 107-122, 1997.

[14] I. Podlubny, "Fractional-order systems and $P I^{\lambda} D^{\mu}$-controllers," IEEE Transactions on Automatic Control, vol. 44, no. 1, pp. 208214, 1999.

[15] W. J. Rugh, Linear System Theory, Prentice Hall, 1996. 
[16] T. Kailath, Linear Systems, Prentice-Hall, Englewood Cliffs, NJ, USA, 1980.

[17] Y. Chen, H.-S. Ahn, and D. Xue, "Robust controllability of interval fractional order linear time invariant systems," Signal Processing, vol. 86, no. 10, pp. 2794-2802, 2006.

[18] J. L. Adams and T. T. Hartley, "Finite-time controllability of fractional-order systems," Journal of Computational and Nonlinear Dynamics, vol. 3, no. 2, Article ID 021402, 2008.

[19] J. Wei, "The controllability of fractional control systems with control delay," Computers \& Mathematics with Applications, vol. 64, no. 10, pp. 3153-3159, 2012.

[20] A. B. Shamardan and M. R. A. Moubarak, "Controllability and observability for fractional control systems," Journal of Fractional Calculus, vol. 15, pp. 25-34, 1999.

[21] K. Balachandran, J. Y. Park, and J. J. Trujillo, "Controllability of nonlinear fractional dynamical systems," Nonlinear Analysis, Theory, Methods \& Applications, vol. 75, no. 4, pp. 1919-1926, 2012.

[22] T. L. Guo, "Controllability and observability of impulsive fractional linear time-invariant system," Computers \& Mathematics with Applications, vol. 64, no. 10, pp. 3171-3182, 2012.

[23] I. Podlubny, Fractional Differential Equations: An Introduction to Fractional Derivatives, Fractional Differential Equations, to Methods of Their Solution and Some of Their Applications, vol. 198, Academic Press, 1998.

[24] H. M. Srivastava and J. J. Trujillo, Theory and Applications of Fractional Differential Equations, Elsevier Science, 2006.

[25] T. Kaczorek, "Positive linear systems with different fractional orders," Bulletin of the Polish Academy of Sciences, vol. 58, no. 3, pp. 453-458, 2010. 


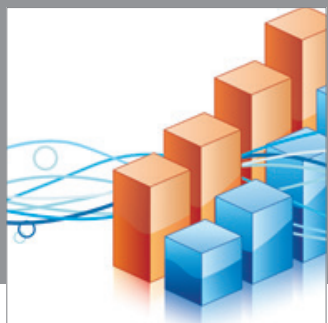

Advances in

Operations Research

mansans

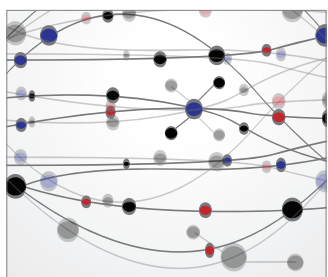

The Scientific World Journal
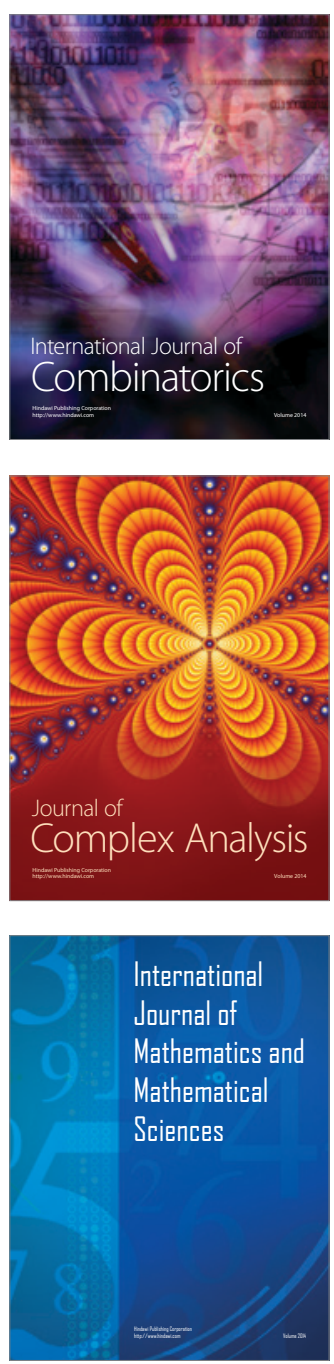
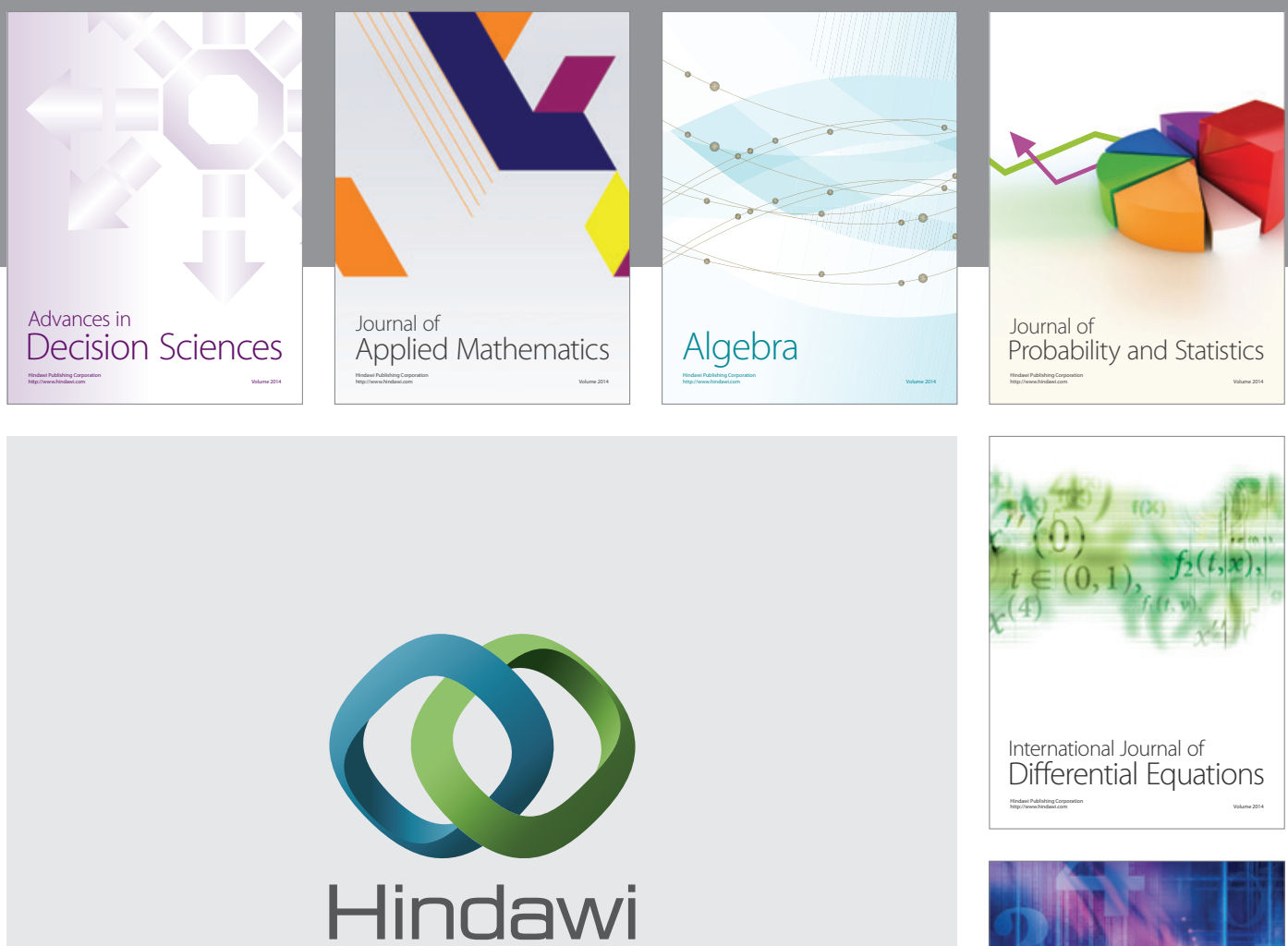

Submit your manuscripts at http://www.hindawi.com
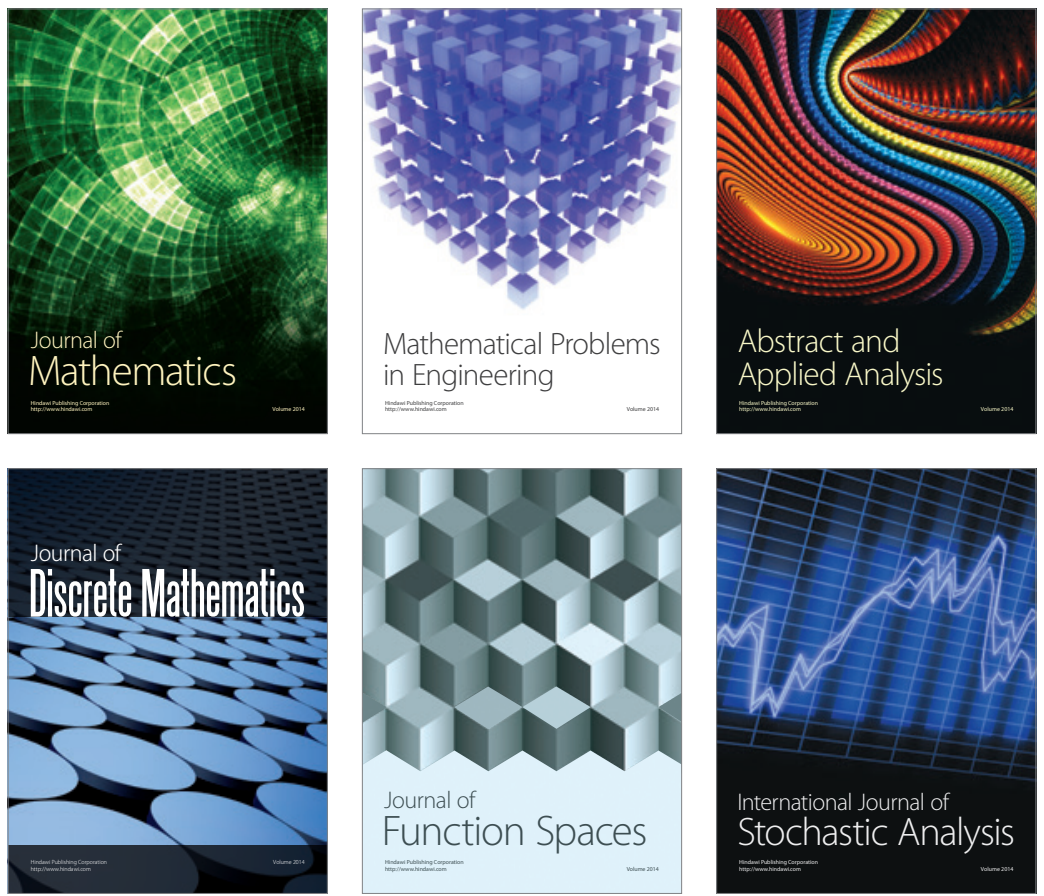

Journal of

Function Spaces

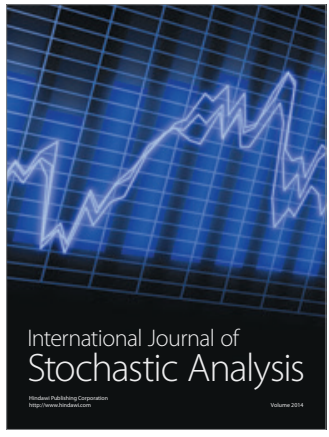

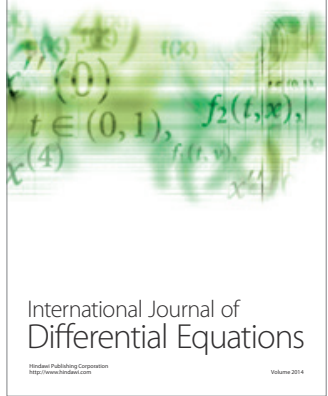
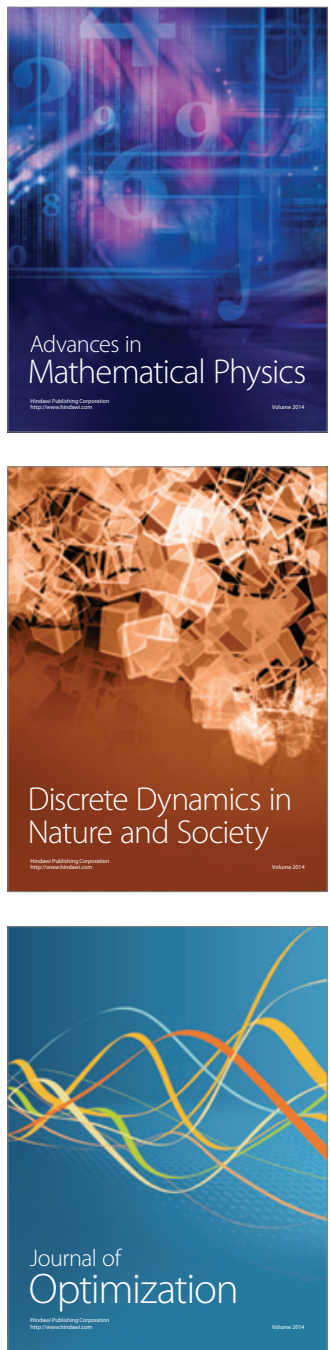